\title{
El apoyo audiovisual en la docencia online durante el estado de alarma: dos experiencias en la Universitat Politècnica de València
}

\author{
Josep Prósper Ribes ${ }^{a}$ y Francisca Ramón Fernández \\ a Profesor titular. Departamento de Comunicación Audiovisual, Documentación e Historia del Arte. Universitat \\ Politècnica de València. jprosper@har.upv.es, ${ }^{b}$ Profesora titular. Departamento de Urbanismo. Universitat Politècnica \\ de València. frarafer@urb.upv.es
}

\section{\$ EWWDFW}

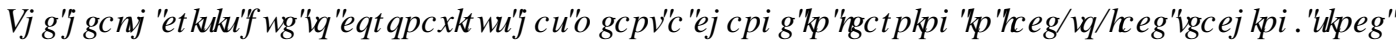

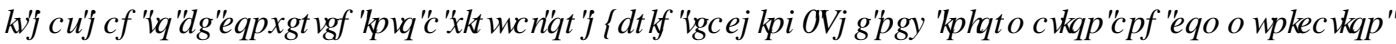

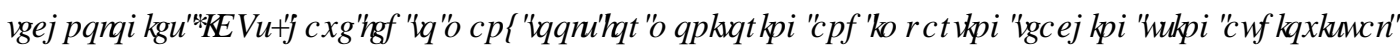

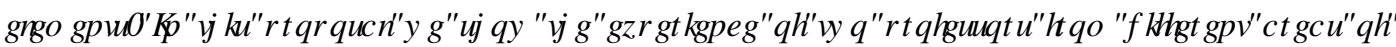

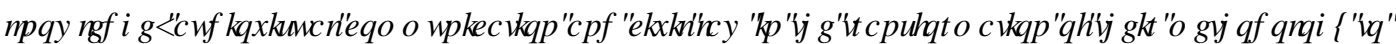

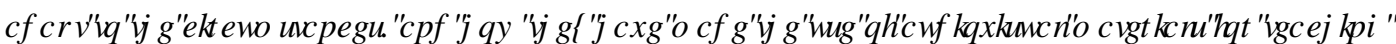

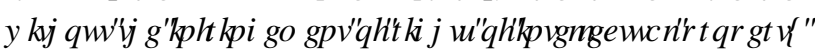

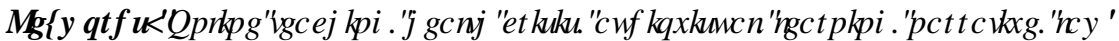

\section{HXP HQ

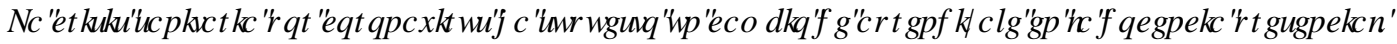

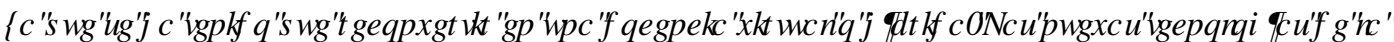

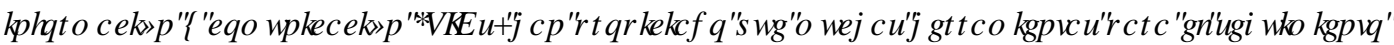

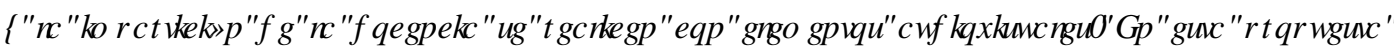

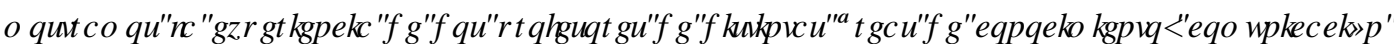

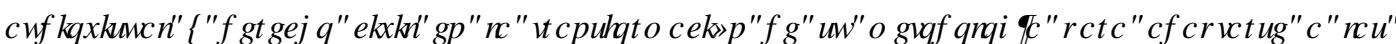

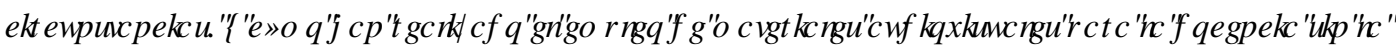

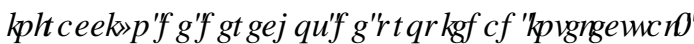

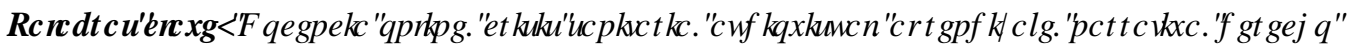

\section{Introducción}

Hay que tener en cuenta que las nuevas tecnologías de la información y comunicación (TICs) tienen una presencia considerable no sólo en la docencia presencial, sino también en la virtual e híbrida (Ramón y Saz, 2004; Canós y Ramón, 2005). No obstante, la docencia virtual o docencia online prescinde de la presencia física en un aula tanto por parte del profesor como del alumnado. Esa presencia física se sustituye por una presencia virtual en la que entran en juego nuevas herramientas para el aprendizaje, seguimiento y evaluación del alumnado.

Durante el periodo de alarma que hemos y seguimos viviendo, que ha afectado al curso 2019-2020 y 20202021, el apoyo audiovisual ha sido fundamental para que profesorado y alumnado pudieran realizar la transmisión de conocimientos y el aprendizaje previsto. 


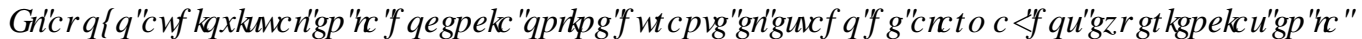 8 QYYHUWDBB RQDAFQIFDTH9DQQRWD}

Muchos de estos elementos audiovisuales que se utilizan en la docencia tienen que observar lo indicado en la normativa de propiedad intelectual, en especial la cita para la docencia e investigación que establece el artículo 32, del Real Decreto Legislativo 1/1996, de 12, de abril, por el que se aprueba el texto refundido de la Ley de Propiedad Intelectual, regularizando, aclarando y armonizando las disposiciones legales vigente sobre la materia, referente a "Citas y reseñas e ilustración con fines educativos o de investigación científica" con la finalidad de no infringir los derechos morales y patrimoniales de autor. Es por ello, que son distintos los medios para la incorporación de contenidos audiovisuales en los recursos destinados a la docencia (Payri, Prósper y Ramón, 2012; Prósper, Payri y Ramón, 2012; Payri, Ramón y Galán, 2013; Payri, Ramón y Blasco, 2014; Payri et al, 2014).

Es habitual que el profesorado genere una serie de recursos audiovisuales para la docencia, principalmente en la modalidad presencial, como apoyo para el aprendizaje, destacando los objetos de aprendizaje, módulos de aprendizaje, MOOC, o cualquier otro elemento que contenga un elemento audiovisual en su diseño (Ramón et al., 2019). Estos recursos también sirven para la evaluación de las competencias transversales de la Universitat Politècnica de València (Ramón et al., 2017, 2018a, 2018b y 2018c), ya que facilitan al profesorado la labor de comprobación del cumplimiento de la misma. Suponen, en definitiva, un recurso para la motivación del alumnado (Lull y Ramón, 2017).

La conversión de la docencia presencial en docencia online durante el estado de alarma ha supuesto la utilización de medios audiovisuals en el aprendizaje de una forma mucho más evidente y con mayor asiduidad que en el escenario presencial. Tanto en la disciplina de Comunicación Audiovisual como de Derecho civil los recursos audiovisuales han sido primordiales para poder lograr el aprendizaje del alumnado.

No solo se han utilizado recursos audiovisuales, sino también medios audiovisuales para la docencia online. De esta forma, podemos destacar la utilización de objetos y módulos de aprendizaje y MOOC (Ramón, 2009, 2010, 2011a, 2011b y 2014), junto con series y películas para complementar los conceptos objeto de las asignaturas a impartir. Y también las plataformas como Teams, que ha supuesto una nueva forma de canalizar la comunicación con el alumnado, a través de la impartición de la docencia mediante una forma síncrona o asíncrona, con la posibilidad de grabar las sesiones docentes. La finalidad es también conseguir la interactuación del alumnado con los materiales (Payri, Blasco y Ramón, 2011; Galán, Ramón y Payri, 2013).

En dicha situación, internet se ha convertido en una plataforma docente (Prósper, Payri y Ramón, 2014).

Se va a mostrar la experiencia docente en la docencia online durante el curso 2019/2020 en tres asignaturas muy diferentes, durante el estado de alarma, en las que el apoyo audiovisual ha sido fundamental para el desarrollo de la docencia:

a) Estudios Fílmicos y Realización de Reportajes Audiovisuales. Grado de Bellas Artes. Facultad de Bellas Artes. 20 alumnos.

b) Derecho civil II. Máster Universitario en Gestión Administrativa. Facultad de Admnistración y Dirección de Empresas. 19 alumnos.

\section{Objetivos}

Los objetivos que vamos a desarrollar son los siguientes:

1- Exponer la necesidad del apoyo audiovisual en las clases on line dado que facilita y permite que el alumno pueda entender mucho mejor los conceptos teóricos y prácticos que componen el contenido de las asignaturas. 
2- Desarrollar las diferentes modalidades de apoyo audiovisual a la docencia, haciendo especial referencia a su utilización en las clases on line.

3- Plantear que el alumno puede y debe participar en la elaboración de material audiovisual, tanto como un ejercicio en determinadas asignatura audiovisuales, como para completar la actividad docente, y de esta manera que no sea un ente pasivo sino activo.

\section{El empleo de medios audiovisuales en Comunicación Audiovisual en la docencia online}

El uso de material audiovisual como apoyo en la docencia de asignaturas de comunicación audiovisual (independientemente del grado en que se impartan), es fundamental para lograr la adecuada comprensión de los alumnos de los contenidos. Es más, podemos decir que sin este apoyo es imposible la adecuada compresión de la materia.

La experiencia que vamos a relatar toma como punto de partida la actividad docente desarrollada en dos asignaturas del grado de Bellas Artes: Estudios Fílmicos y Realización de Reportajes Audiovisuales. Sin embargo, conviene considerar que se parte de la experiencia docente que el profesor tuvo en otras asignaturas impartidas en la antigua licenciatura de Comunicación Audiovisual.

Vamos a considerar tres modalidades:

1- Uso de fragmentos de obras audiovisales durante las clases on-line.

2- Creación de material audiovisual donde se exponen conceptos y contenidos.

3- Realización de trabajos audiovisuales por parte de los alumnos.

A continuación paseremos a exponer cada una de estas modalidades.

\subsection{Uso de fragmentos de obras audiovisuales durante las clases online}

Los fragmentos de producciones audiovisuales se utilizan para ejemplificar los conceptos expuestos en las clases teóricas de forma que el alumno pueda entender con más facilidad los contenidos, al mismo tiempo que comprueba su utilización práctica. En este sentido, no hay mucha diferencia aparente con respecto a la clase presencial. Pero al poder reproducir la clase grabada y poder descargar al mismo tiempo en su ordenador la señal del archivo audiovisual, se facilita enormente su posterior visionado y estudio para poder entender mejor los conceptos expuestos (Fig. 1). Hay que tener en cuenta que estos archivos audiovisuales pueden cumplir una doble función:

-A Complementar la explicación del profesor.

-B Constituir por sí mismos conceptos fundamentales por ser fuente de ideas y conocimientos. 


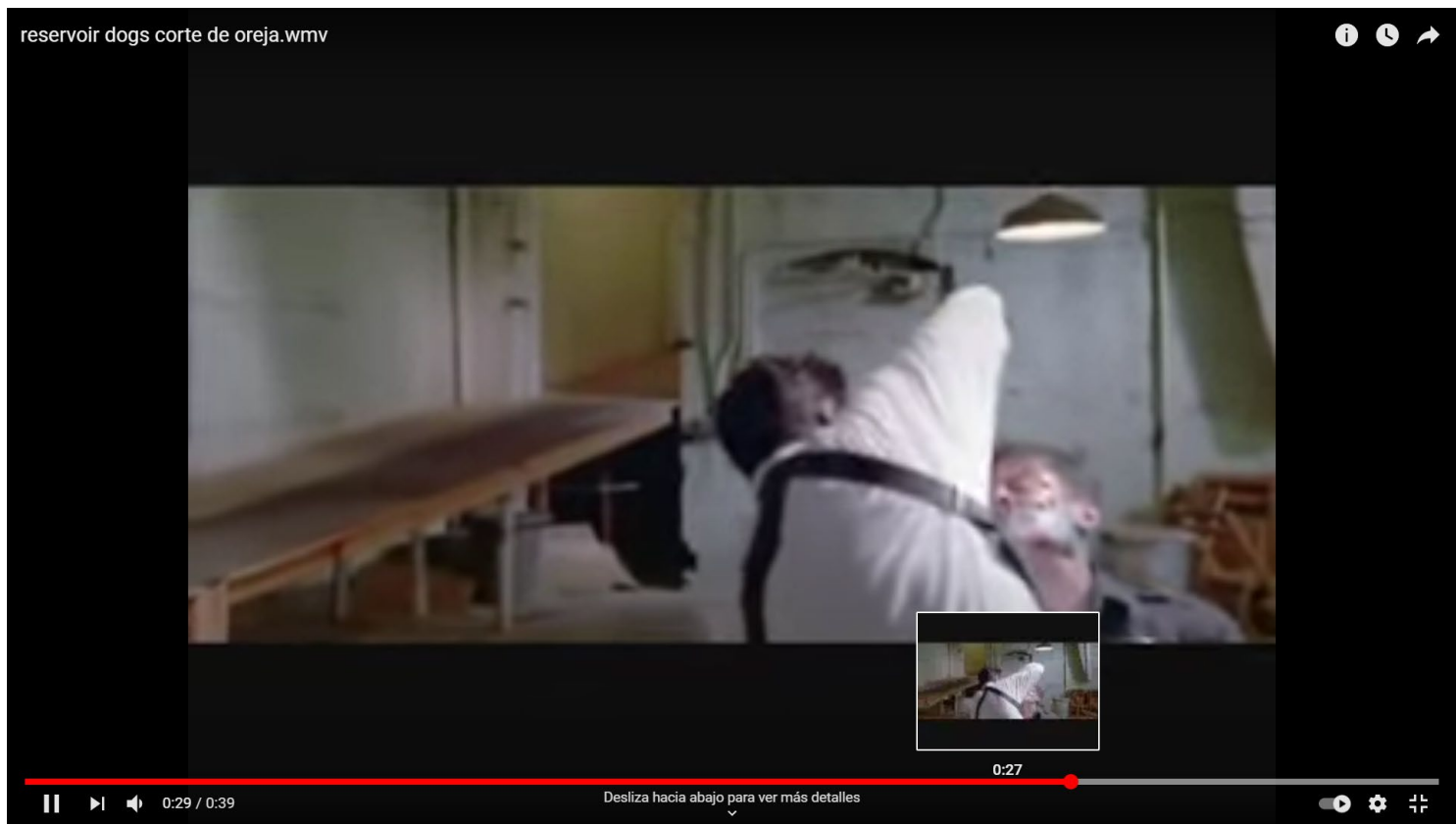

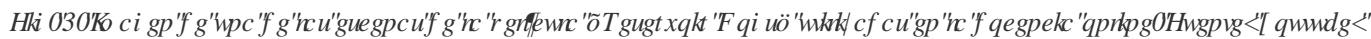
KWSVIZZZロ RXWEHFRP ZZDWK'Y 3GP\%\& L50 ( प

\subsection{Creación de material audiovisual donde se exponen conceptos y contenidos}

En este caso, se produce un material grabado para que el alumno pueda visionarlo posteriormente de forma libre. Es tanto el caso de los MOOCs (Prósper y Ramón, 2019), como de la creación bajo la dirección del profesor de producciones audiovisuales de corta duración por parte de los alumnos (bien individual, bien grupal) que desarrollen conceptos y contenidos de la asignatura. Por supuesto, estos archivos también se pueden añadir a una plataforma on line de forma que el alumno pueda acceder a ellos libremente.

\subsection{Realización de trabajos audiovisuales por parte de los alumnos}

Creación de materiales por parte de los alumnos como complemento a su formación. Es este caso, también es una forma de evaluación. El control del profesor se puede realizar de forma on line, como así ha ocurrido en la asignatura Realización de Reportajes Audiovisuales del curso 2019/20, donde los alumnos desde sus respectivos lugares de residencia (ya fuera en España o en otros países como Italia o la República Checa) realizaban dentro de las limitaciones impuestas por la pandemia las grabaciones originales y por videoconferencia las mostraban al profesor comentando las diferentes particularidades. Posteriormente, se realizaban los trabajos necesarios para su elaboración final, como redacción del off final y edición de las imágenes visuales y auditivas. Todo este proceso era controlado por el profesor a través de videoconferncia con los alumnos, y se analizaba todo el proceso fase a fase hasta la producción final. También hubo alumnos que no pudieron realizar una grabación original. En este caso, se recurrió a la realización de reportajes de archivo, es decir, a tomar imágenes de otras producciones a través de Internet y a partir de ahí, realizar el reportaje. Fue una experiencia muy compleja, pero satisfactoria. Se demostró que el uso de Internet era muy válido para poder plantear una asignatura tan compleja como Realización de Reportajes Audiovisuales, donde el trabajo de creación de los alumnos es fundamental (Fig 2). 


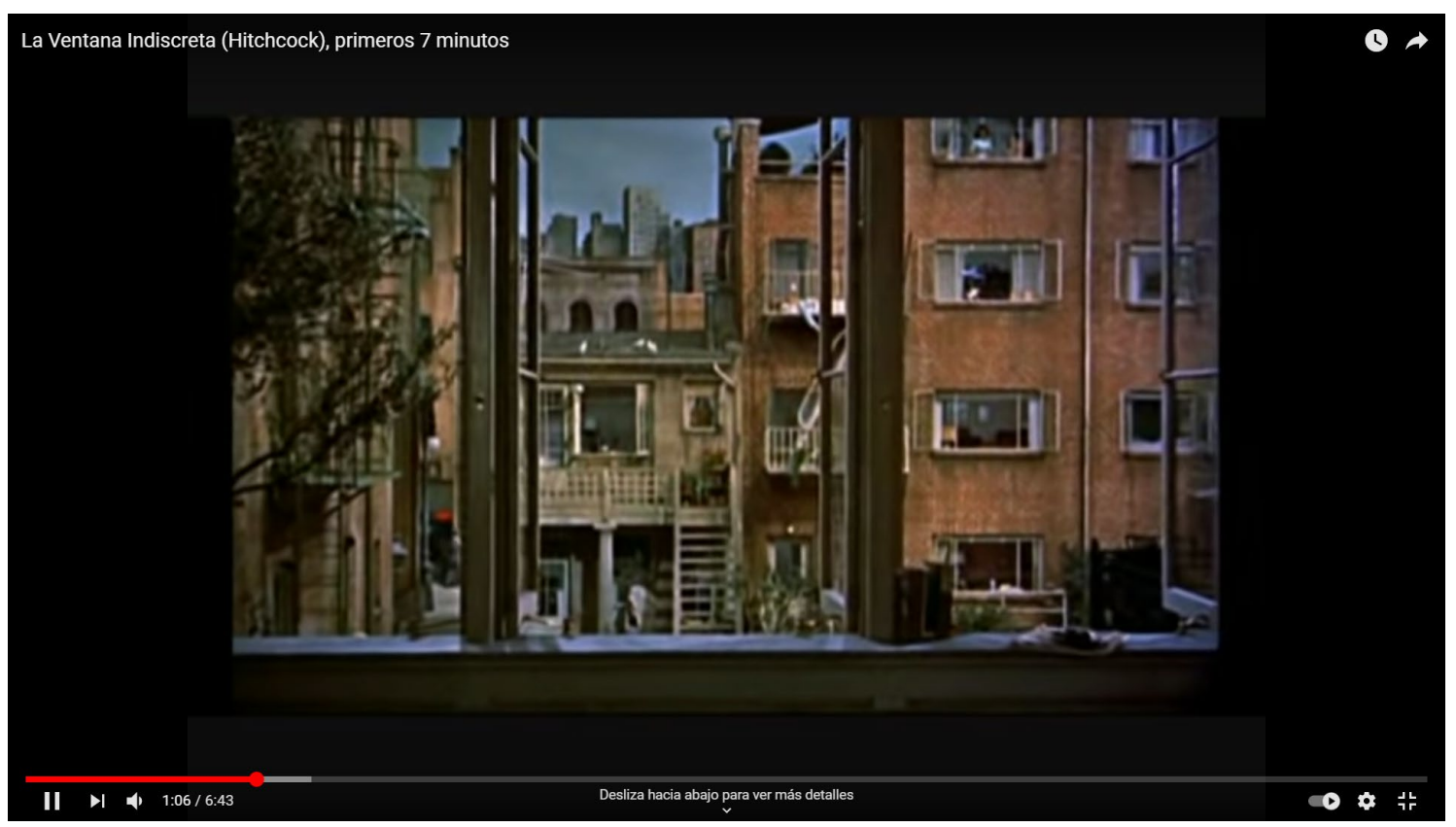

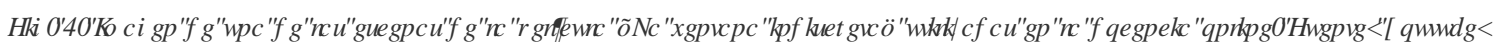

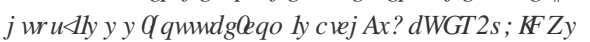

\section{El empleo de medios audiovisuales en Derecho civil en la docencia online}

Vamos a mostrar la experiencia de la utilización de medios audiovisuales en la asignatura de "Derecho civil II”, que se impartió en el Máster Universitario en Gestión Administrativa, durante el curso académico 20192020, con un total de 19 alumnos.

Esta asignatura comenzó durante el mes de febrero del año 2020, y coincidió con la declaración del estado de alarma, cuando se habían impartido solamente unas pocas clases, ya que se imparte una sesión de tres horas a la semana, y la última clase presencial fue el 11 de marzo de 2020, apenas unos días antes de la declaracion del estado de alarma.

En esta asignatura se utilizaba el sistema de videoapuntes (implantado hace tiempo en la Universitat Politècnica de València, tras el Proyecto Opencast Matterhorn) (Ramón, 2012; Ramón, Payri y Prósper, 2012), siendo una herramienta de gran utilidad para el alumnado, ya que le permitía "repasar" los conceptos una vez finalizada la clase (Fig. 3).

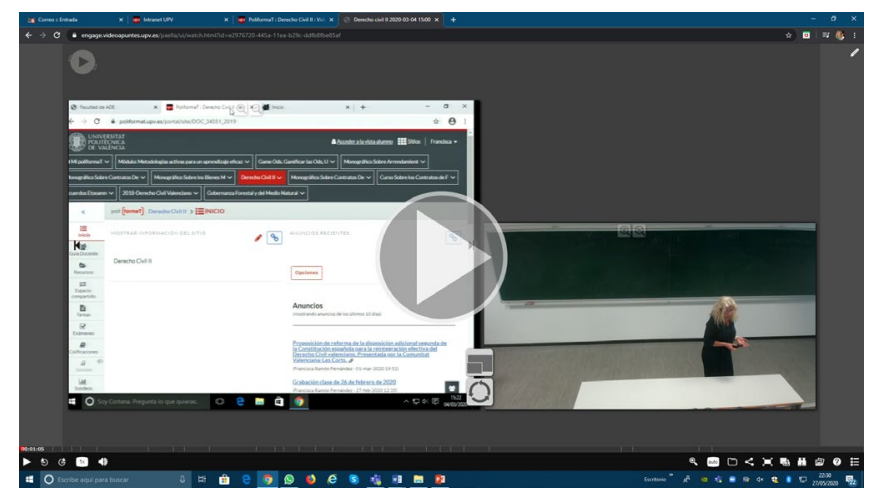

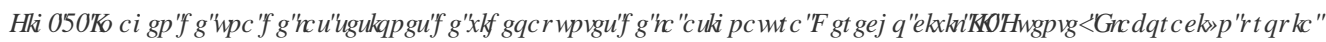

(cc) BY-NC-ND 2021, Universitat Politècnica de València

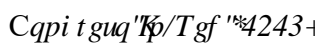


A partir de dicho momento, se tienen que realizar una serie de adaptaciones de la docencia que consistieron en lo siguiente:

-Adaptación de la guía docente. Se tiene que acondicionar a la situación de docencia virtual, y determinar qué recursos se han a utilizar en la docencia, teniendo en cuenta que la docencia ya había comenzado, y se tiene que realizar la adaptación de forma inmediata.

-Elaboración de materiales para el alumnado. Se seleccionan y se elaboran de nuevo materiales para que el alumnado pueda seguir la asignatura, de ahí la reconversión de materiales para la impartición de la docencia online, por ejemplo, a través del diseño de test, que permitieran al alumnado una retroalimentación de conocimientos.

-Actividades para evaluar. Las tareas habituales se rediseñan para que puedan realizarse de forma virtual, con exposiciones virtuales, y participación online.

-Establecimiento de canales de contacto con el alumnado. Se utiliza teams, como canal principal de contacto, y de apoyo y ayuda al alumnado.

-Disposición para el alumnado de materiales complementarios. Se le facilitan artículos de prensa, artículos científicos como refuerzo para la consolidación de conocimientos. Se empieza a trabajar de forma virtual en vez de presencial, con todas las herramientas accesibles por internet.

Para ello, se realizan las siguientes actuaciones:

-Diseño de 25 tests autocorregibles (Fig. 4).

-Realización de un modulo de aprendizaje.

-Disposición al alumnado de material complementario a través de un curso MOOC de acceso abierto (Fig. $5)$.

-Facilitar distintos objetos de aprendizaje como refuerzo del aprendizaje de conceptos.

-Utilización de teams como medio de comunicación con el alumnado (Fig. 5).

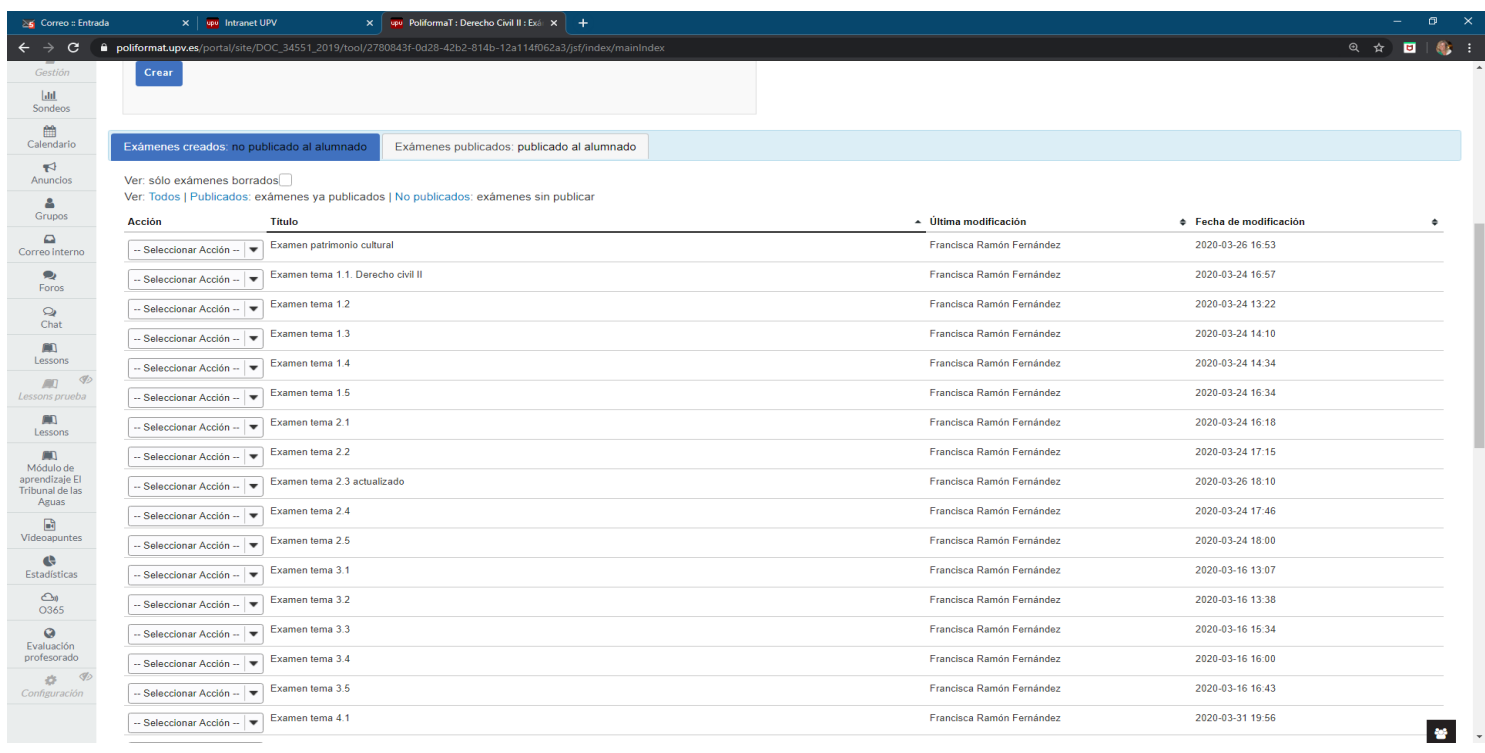

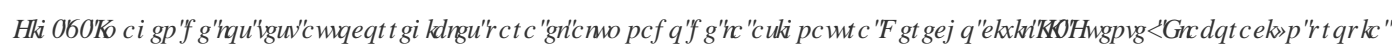




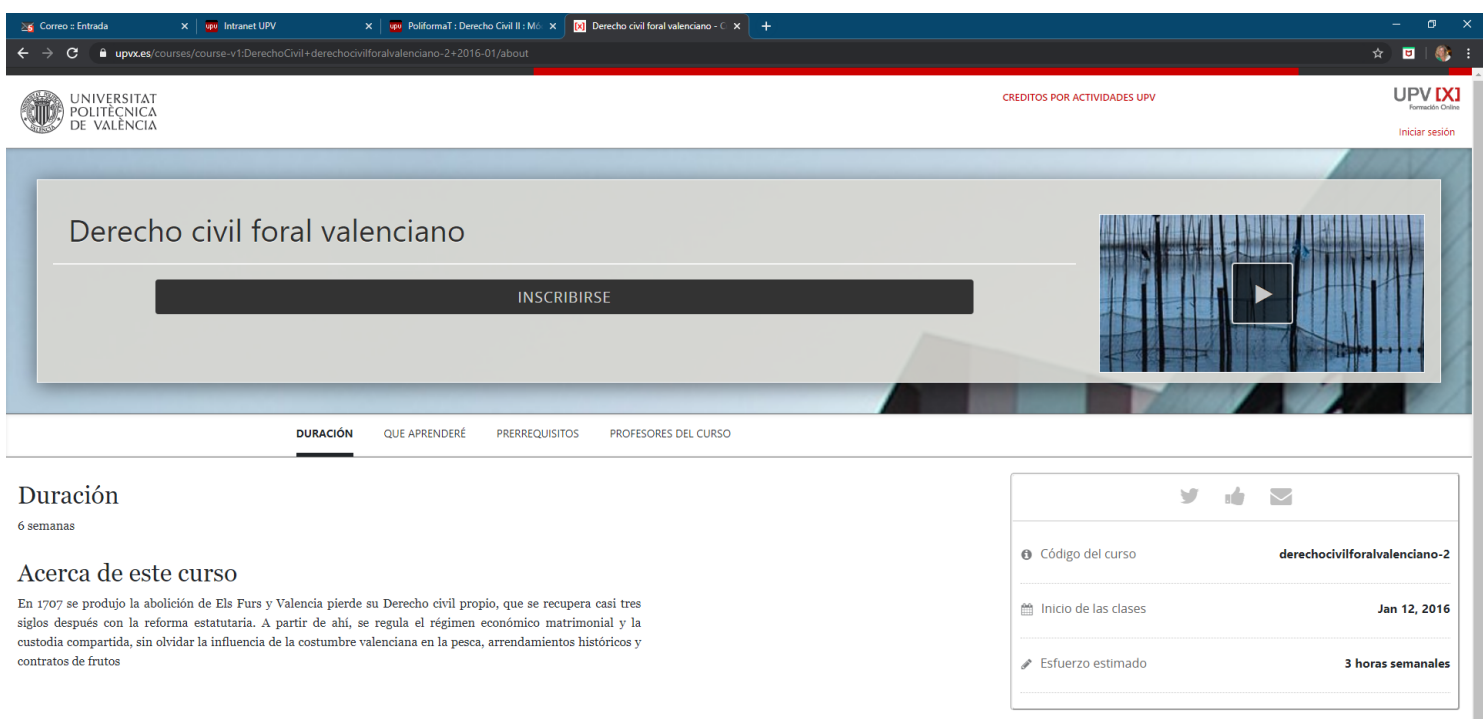

f 8 :

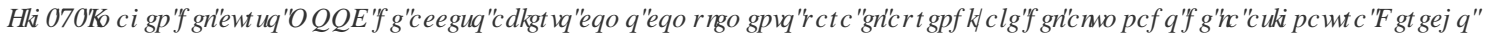
FIYQ, ए) XHQUAL( QERLFIYQSLRSID]

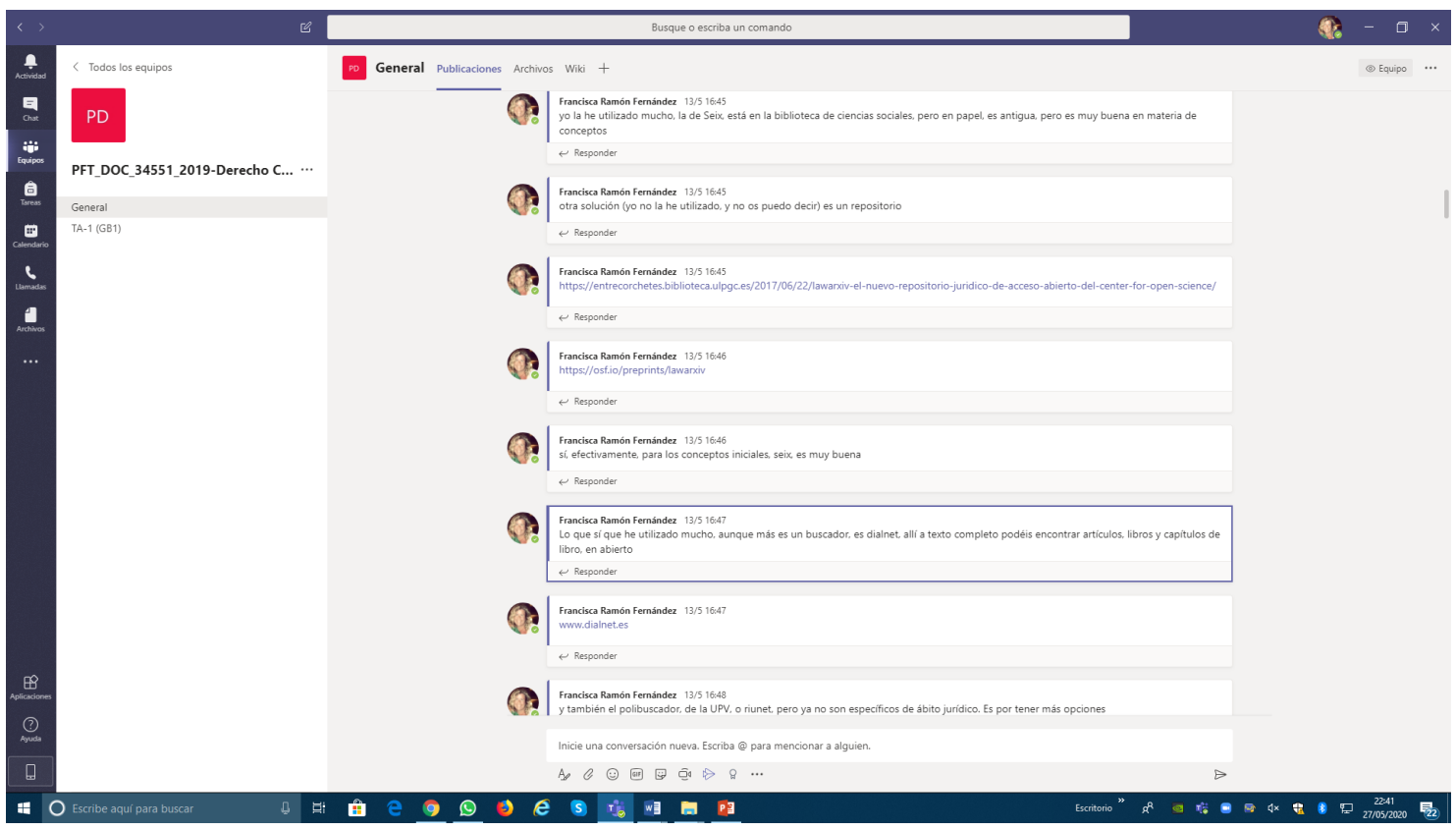

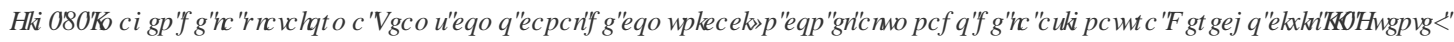
( QERLEFIYQSLRSID]

La eliminación de la presencialidad durante el periodo del estado de alarma supuso un periodo de adaptación de forma inmediata tanto para el profesorado como para el alumnado. No hubo tiempo para la creación de los nuevos materiales, ya que se tuvieron que diseñar y elaborar de forma inmediata. Se reutilizan materiales ya elaborados, y también se le facilita al alumnado mucho más material complementario que en la docencia presencial, como complemento para su formación.

Las ventajas de la impartición de la docencia online fueron la extraordinaria disposición del alumnado que se adaptó sin ningún problema a la modalidad no presencial; matenimiento permanente del contacto con el 


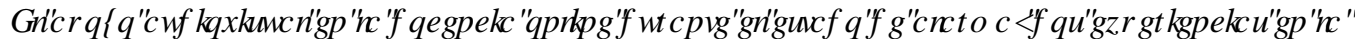 8 QYYHUWDBB RQDAFQIFDTH9DQQRWD}

alumnado; la obtención de resultados similares a la presencialidad, no hubo diferencias, ya que exceptuando el test presencial que se sustituyó por los test online, las prácticas se elaboraron de la misma forma, e incluso la exposición la realizaron online los alumnos.

Como inconvenientes destacamos: la saturación de la información; la no desconexión en ningún momento (Ramón, 2021), y las dificultades del alumnado para acceder a internet (Ramón, Lull y Soriano, 2020).

Los resultados obtenidos fueron altamente positivos. De los 16 alumnos matriculados, 12 hicieron el seguimiento de forma óptima, siendo 4 alumnos los que no realizaron ningún seguimiento, ni tuvieron contacto ni al inicio de la docencia, cuando era en la modalidad presencial.

La media obtenida por los alumnos fue de sobresaliente en las actividades y en los test autocorregibles.

De igual modo, la encuesta docente realizada al alumnado, recogiéndose 11 respuestas de los 12 que siguieron la asignatura, mostraron su satisfacción general con la forma de resolver la situación durante el periodo de confinamiento, siendo la valoración de 9.09.

\section{Conclusiones}

La adaptación sin solución de continuidad de la docencia presencial a la docencia virtual durante el periodo del estado de alarma supuso un reto para alumnado y profesorado.

Para el profesorado supuso un aumento de la carga de trabajo considerable, además de no disponer de desconexión digital, la falta de delimitación de un horario para las consultas, y la necesidad de utilizar plataformas y herramientas audiovisuales que no había utilizado con anterioridad.

Para el alumnado supuso un cambio de escenario "a mitad del partido" de forma inmediata, y teniendo que reconvertirse la evaluación en determinados casos, así como la utilización también de plataformas que no había utilizado con anterioridad, con lo que se generaba

Hay varios tipos de apoyo audiovisual que se pueden utilizar con finalidad docente en las clases on line, y ello debe ser tenido en cuenta a la hora de la planificación de la docencia en una situación de docencia virtual. Cada una de estas modalidades tiene unas posibilidades concretas, aunque el fin último es siempre facilitar la comprensión de los contenidos.

Ello se pone de manifiesto, pues, que el apoyo audiovisual para las clases online ha resultado imprescindible y ha facilitado la comprensión de los conceptos, siendo uno de los objetivos de la inclusión de dicho apoyo en la docencia.

Básicamente, podemos hacer una gran división: material elaborado directamente por el docente y materiales elaborados por los alumnos bajo la dirección de los profesores. En este último tipo, se pueden considerar los siguientes supuestos:

1- Ejercicios de carácter audiovisual donde el alumno desarrolla una técnica o procedimiento narrativo concreto.

2- Ejercicios destinados a completar los conceptos teóricos y facilitar su comprensión.

Este apoyo audiovisual se ha puesto de manifiesto en distintas asignaturas, que han utilizado diversas herramientas en la docencia, cumpliendo el objetivo de que es preciso el apoyo audiovisual en la docencia online.

La valoración de la experiencia es positiva y se ha solventado de forma favorable facilitando el aprendizaje a través de la utilización de medios audiovisuales ya conocidos y nuevos que se han utilizado por primera vez en la docencia online. 
Plantear que el alumno puede y debe participar en la elaboración de material audiovisual, tanto como un ejercicio en determinadas asignatura audiovisuales, como para completar la actividad docente, y de esta manera que no sea un ente pasivo sino activo.

\section{Agradecimientos}

Trabajo realizado en el marco del Proyecto de Innovación y Mejora Educativa (PIMEs) "Gamificación y TICs: diseño de actividades audiovisuales basadas en la ciencia ficción para la dinamización docente en un entorno presencial, semipresencial y virtual", presentado en el marco de la convocatoria de Proyectos de Innovación y Mejora Educativa (PIMEs) llevada a cabo en la Universitat Politècnica de València para el curso 2020-2022 obteniendo resolución favorable de la Comisión de Evaluación y Seguimiento de Proyectos de Innovación y Convergencia (CESPIC) en su sesión de septiembre de 2020 y concedido por el Vicerrectorado de Estudios y Convergencia Europea de la Universitat Politècnica de València. Años 20202022. Investigadora principal: Francisca Ramón Fernández. y en el marco del Proyecto I+D+i «Retos investigación» del Programa estatal de $\mathrm{I}+\mathrm{D}+\mathrm{i}$ orientado a los Retos de la Sociedad del Ministerio de Ciencia, Innovación y Universidades: RTI2018-097354-B-100. «Contratos, transparencia y protección de datos en el mercado digital» (2019-2022). Investigadores Principales: Dr. Javier Plaza Penadés, Catedrático de Derecho Civil, y Dra. Luz M. Martínez Velencoso, Catedrática de Derecho Civil. Universitat de València-Estudi General.

Los autores han contribuido en la misma medida en la elaboración del presente trabajo. El orden de firma ha sido determinado exclusivamente siguiendo el criterio alfabético del primer apellido de cada autor.

\section{Referencias}

CANÓS DARÓS, L. y RAMÓN FERNÁNDEZ, F. (2005). “Aplicación de las nuevas tecnologías para el trabajo

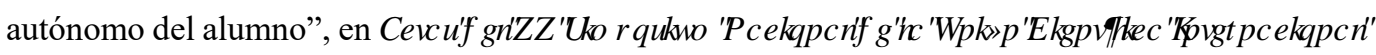

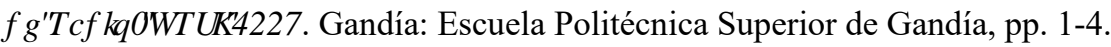

GALÁn Cubillo, E., RAMÓn Fernández, F. y PAYRI, B. (2013). “La utilización de estrategias interactivas en el proceso de gestación del relato audiovisual con fines docentes", en $3 D W X P D D) R[P D O m R]$

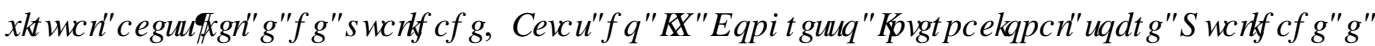

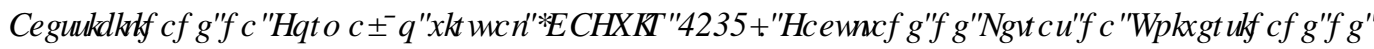

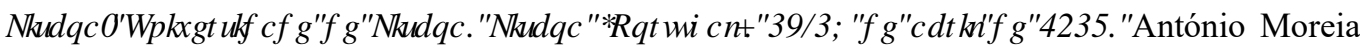
Teixeira, Luis Bengoechea, José Ramón Hilera (Editores). Lisboa-Madrid: Facultade de Letras, Universidade de Lisboa y Universidad de Alcalá, pp. 436-444.

$<$ http://www.esvial.org/cafvir2013/documentos/LibroActasCAFVIR2013.pdf $>$ [Consulta: 20 de enero de 2021].

PAyri, B., Blasco YePes, C. y RAMÓn Fernández, F. (2011). "La interactividad para la mejora de los test con material audiovisual”, en , , -RLQDODVGH, QQRVDFIy Q GHD, QMUXWR GH \&IHQFDV GH (D)]

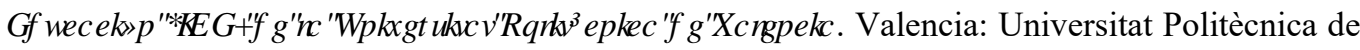
València.

PAYRI, B., PRÓSPER RIBES, J. y RAMÓn FERnÁNDEZ, F. (2012). “Comparativa de sistemas para introducir

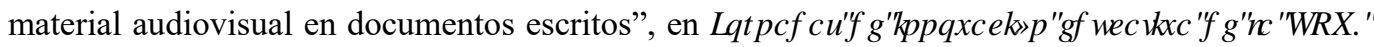

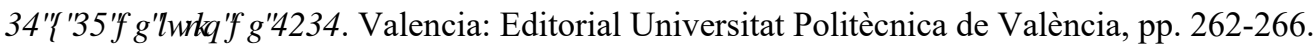

Payri, B., Ramón Fernández, F. y Blasco Yepes, C. (2014). "Creación e inserción de materiales audiovisuales en un sitio web para la docencia de música y sonido audiovisual”, en ,' ( 6००००ण 


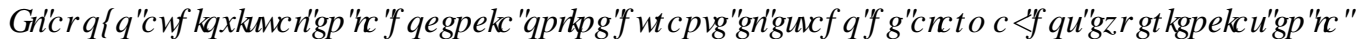

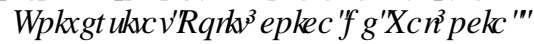

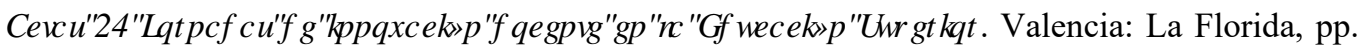
183-187.

PAyri, B., RAmón Fernández, F. y Galán Cubillo, E. (2013). "Inserción de ejemplos musicales y audiovisuales en línea para la docencia sobre sonido", en 3DDXP D) RLP DOTRTYLWDDODFHAYHDH

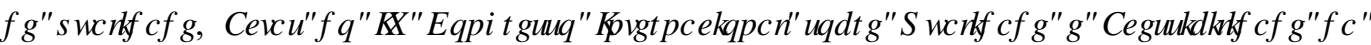

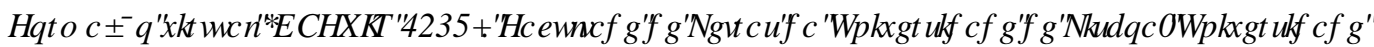

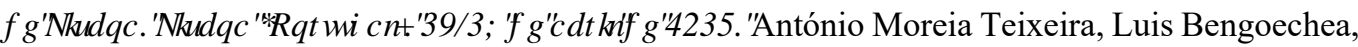
José Ramón Hilera (Editores), Lisboa-Madrid: Facultade de Letras, Universidade de Lisboa y Universidad de Alcalá, pp. 420-428.

$<$ http://www.esvial.org/cafvir2013/documentos/LibroActasCAFVIR2013.pdf $>$ [Consulta: 20 de enero de 2021].

PAYRI, B. et al. (2014). "Artículos docentes de estudios audiovisuales ilustrados con material audiovisual: adaptación a las normas del ICE-UPV”, en - RLQDODVGHIQRYDFIYQHCXFDUMDI IGRFHQIDHQUHE प,1 प5( ' क्ष००. Valencia: Universitat Politècnica de pp. 181-194.

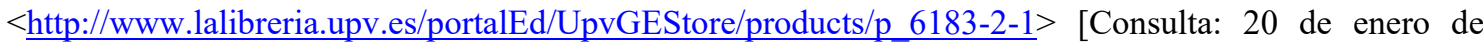
2021].

PrósPer RiBes, J. y RAMÓN FERnÁndeZ, F. (2012). "MOOC y derechos de autor en la enseñanza online

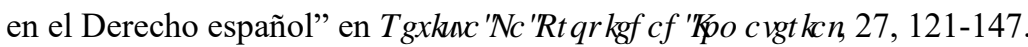

$<$ https://revistas.uexternado.edu.co/index.php/propin/article/view/6075/7793 $>$ [Consulta: 25 de enero de 2021].

Prósper Ribes, J., PAyri, B. y RAmón Fernández, F. (2012). "La enseñanza teórica y práctica del eje

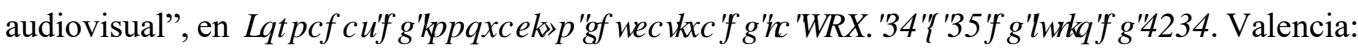
Editorial Universitat Politècnica de València, pp. 295-298.

Prósper Ribes, J., Payri, B. y Ramón Fernández, F. (2014). "El uso de internet como plataforma

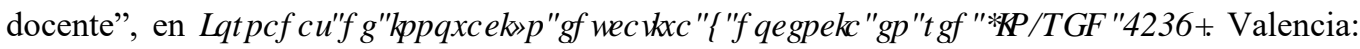
Universitat Politècnica de València, pp. 140-150. $<$ http://www.lalibreria.upv.es/portalEd/UpvGEStore/products/p_6183-2-1> [Consulta: 21 de enero de 2021].

RAMÓN FERNÁNDEZ, F.: y SAZ GIL, Ma . I. (2004). "Las nuevas tecnologías como elemento facilitador de los métodos activos en la transmisión de competencias", en + DFWDXQD GFHQFD GHFDOCDCAD

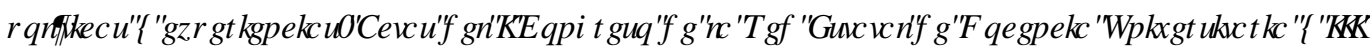

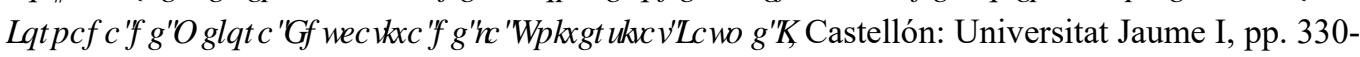
341.

RAMÓN FERNÁNDEZ, F. (2009). "La utilización de los objetos de aprendizaje como nueva tecnología aplicada a la enseñanza técnica de la biotecnología. Su aplicación en la asignatura de Protección Jurídica de la Invención Biotecnológica", en -RLQDDD GH, QQRYDFlyQ ' RFHQUI HQ QD ( VEXHDD 7pFQFD 6XSHURUGH, QJHQHHR \$ J U QRPRV. Valencia: Universitat Politècnica de València, pp. 181-185.

RAMÓN FERNÁNDEZ, F. (2010). "La utilización de los objetos y módulos de aprendizaje en la plataforma virtual poliformat como herramienta para la dinamización de una asignatura", en ; 9, , , $-R L Q D D D V$

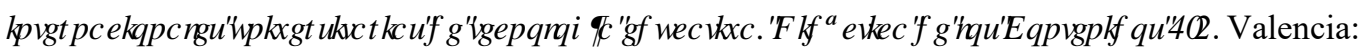
Universitat de València, pp. 1-12. 
RAMÓN FERNÁNDEZ, F. (2011a). "El módulo de aprendizaje: la jerarquía normativa como método para explicar conceptos constitucionales en las Ingenierías", en /D HQMKDQ]D GHD ' HHFKR

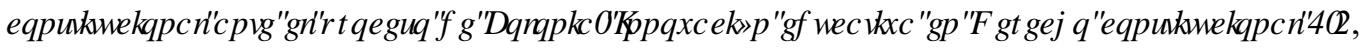
Cotino Hueso, Lorenzo y Presno Linera, Miguel (eds.). Valencia: Publicaciones de la Universidad de Valencia, pp. 310-319. < http://www.uv.es/innova/libroinnovacion2010.pdf $>$ [Consulta: 21 de enero de 2021].

RAMÓN FERNÁNDEZ, F. (2011b). "Módulo de aprendizaje sobre la jerarquía normativa utilizado en la Universidad Politécnica de Valencia", en / DHQMKDQIDGHD HHFRRFRMUXFIRQDODQMAHOSLRFHR]

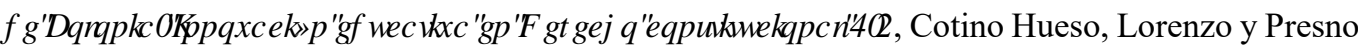
Linera, Miguel (eds.). Valencia: Publicaciones de la Universidad de Valencia, pp. 353-359.

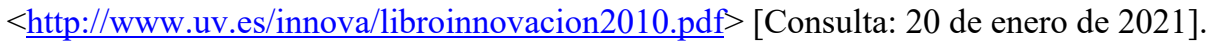

RAmÓn Fernández, F. (2012). "La experiencia del Proyecto Piloto de almacenamiento de clases magistrales utilizando la herramienta Opencast Matterhon en la Universitat Politècnica de

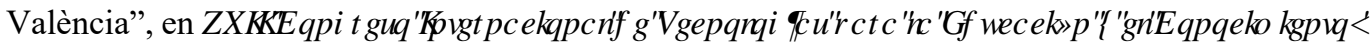
7HFC36RRITDV( P HJ HQWW. Madrid: UNED, pp. 1-13.

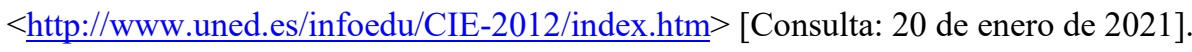

RAMÓn Fernández, F., PAYRI, B. y PRÓSPer RiBES, J. (2012). "La grabación de clases magistrales con Opencast Matterhorn: aplicación docente y marco legal", en - RLQDGDVGHLQRYDFIyQHGXFDUMDGH

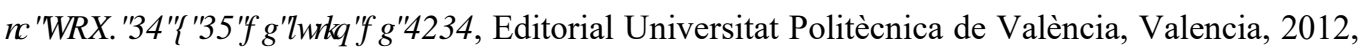
págs. 304-308.

RAMÓN FERNÁNDEZ, F. (2014). "La utilización de los módulos de aprendizaje en las asignaturas jurídicas:

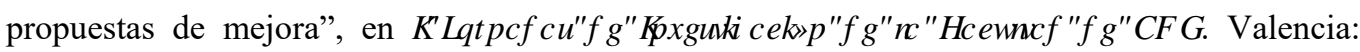
Universitat Politècnica de València, pp. 112-119.

RAMÓN FERNÁNDEZ, F. et. al. (2017). "La utilización de series de ficción para la evaluación de la competencia transversal pensamiento crítico. Análisis de una experiencia en la Universitat

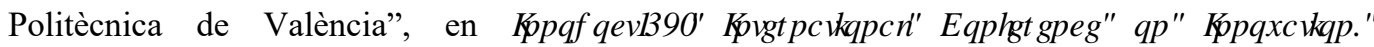
' RFXP HQUMRQDQCE( GXFDURQ Valencia: Universitat Politècnica de València, pp. 377-386.

$<$ https://riunet.upv.es/handle/10251/107064> [Consulta: 20 de enero de 2021].

Lull Noguera, C. y RAMÓn FernÁndez, F. (2017). "Uso de herramientas digitales para mejorar la

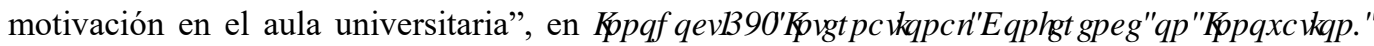

' RFXP HQMUARQDQCF( GXFDURQ. Valencia: Universitat Politècnica de València, pp. 387-397.

$<$ https://riunet.upv.es/handle/10251/107064> [Consulta: 20 de enero de 2021].

RAMÓN FERNÁNDEZ, F. et al. (2018a). "El audiovisual Tutankamon como herramienta para la evaluación de la competencia transversal de pensamiento crítico y responsabilidad ética, medioambiental y

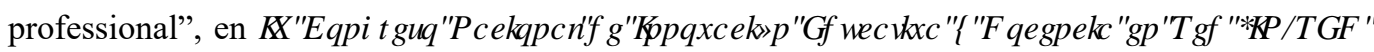
पि००- Valencia: Universitat Politècnica de València, pp. 1-12.

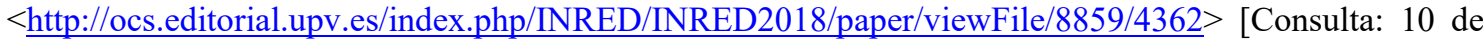
enero de 2021].

RAMÓN FERNÁNDEZ, F. et. al. (2018b). "La utilización de los documentales como herramienta para la evaluación de la competencia transversal CT07. Responsabilidad ética, medioambiental y profesional. Experiencias en la docencia impartida en la Universitat Politècnica de València”, en

(c)) BY-NC-ND 2021, Universitat Politècnica de València

CRQJUMR, Q5 HGDपिएव 


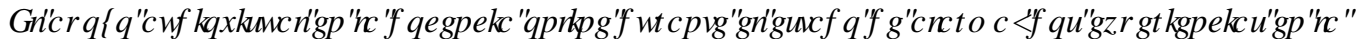 8 QYYHUWDBB RQDAFQIFDTH9DQQRWD}

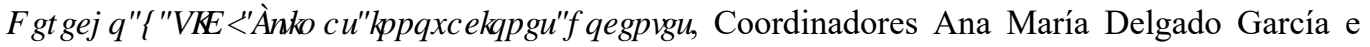
Ignacio Beltrán de Heredia Ruiz. Barcelona: Huygens editorial, pp. 193-204.

RAMÓN FERNÁNDEZ, F. et al. (2018c). "El audiovisual como medio para la aplicación del alumno de la competencia transversal responsabilidad ética, medioambiental y profesional en la Universitat

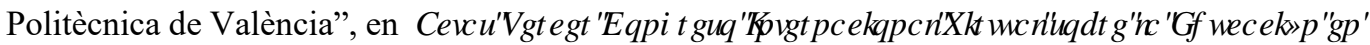
HOMJ RI]; ; , . Málaga: Eumed, pp. 410-429.

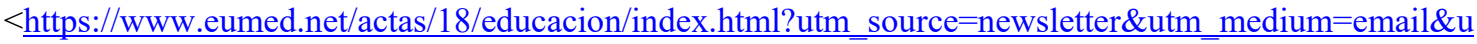
$\underline{\text { tm_campaign }=\text { actas_del_iii_congreso_online internacional_sobre_la_educacion_en_el_siglo_xx }}$ i\&utm term=2018-06-01> [Consulta: 20 de enero de 2021].

RAMÓN FERNÁNDEZ, F. et al. (2019). "Diseño de materiales docentes basados en recursos audiovisuales de humor para la mejora del aprendizaje y su evaluación en ciencias sociales y jurídicas. Análisis del diseño de la actividad sobre las casas Cueva y "Los Picapiedra"”, en / DQRFHQWDGHD HHFKRIHQ QDURFHHDCEUJLMOBarcelona: Huygens Editorial, pp. 35-46.

RAmón Fernández, F., Lull Noguera, C. y Soriano Soto, Ma. D. (2020). "Estrategias de la docencia online y su evaluación durante la crisis sanitaria: recursos y métodos utilizados en la Universitat

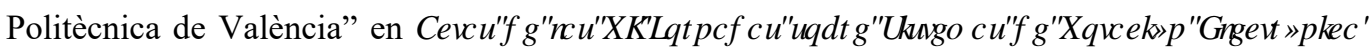

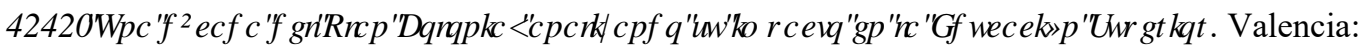
Universitat de València, pp. 10-13.

$<$ https://pages.uv.es/piclickers/images/Actas_VIJSVE2020.pdf?fbclid=IwAR3TgJWeW_NoloZF8VY2R 9urM2cEvEm4vOXLRfQduA01J4p0OaV5RFtf7tQ> [Consulta: 20 de enero de 2021].

RAMÓN FERNÁNDEZ, F. (2021). "La desconexión digital y docencia universitaria online en tiempos de

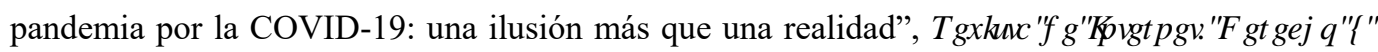
3 ROUFD, núm. 32, pp. 1-15.

$<$ https://www.raco.cat/index.php/IDP/article/view/373744/468833 $>$ [Consulta: 21 de enero de 2021]. 